\title{
MATHEMATICAL MODEL OF A QUEUING SYSTEM WITH ARBITRARY QUANTITY OF SOURCES AND SIZE-LIMITED QUEUE
}

\author{
Alexander Kirpichnikov ${ }^{1}$, Anton Titovtsev ${ }^{2} \S$ \\ ${ }^{1}$ Kazan National Research Technological University \\ 68, K. Marksa, Kazan, 420015, RUSSIA \\ ${ }^{2}$ Kazan National Research Technological University \\ 68, K. Marksa, Kazan, 420015 RUSSIA
}

\begin{abstract}
The paper presents a mathematical model of an open multi-channel queuing system having $m$ service facilities of identical efficiency with exponentially distributed service time. The input stream of Poisson character includes demands of different types arriving from an arbitrary quantity of sources $h$ and having various size-limited queues. General mathematical formulae for probabilistic characteristics, first and second moments of numerical characteristics specifying the quality of service in a steady-state mode of work have been obtained.
\end{abstract}

AMS Subject Classification: $60 \mathrm{~K} 25$

Key Words: queue, quality of service (QoS), queuing system, M/M/m/K, service facility

\section{Introduction}

Issues of studying combined models of queuing originate from Cohen's works (Cohen J.W.) [2], where the combination of Erlang models and classical queuing system was considered for the first time. A number of formulae for probabilities of queuing system (QS) steady states, call loss probability, and first moments

Received: December 16, 2015

Published: February 19, 2016

$\S_{\text {Correspondence author }}$ (c) 2016 Academic Publications, Ltd.

url: www.acadpubl.eu 
of demands number in a queue and waiting time in a queue are given in the paper.

Another specific case of a combined model is a mixed system with losses and expectation having some servers and finite memory, presented in the work of H. Takagi [6]. In this case there are two sources of demands in the system, thus demands from the first source will be lost if all servers are busy at the time of their arrival in the system. Demands from the second source are accepted in a queue only if the number of demands in it does not exceed some defined value K. Streams of demands arriving in the system also have a Poisson character. Formulae for probabilistic characteristics of the system and for the moments of $n$ order of waiting time and common delay time in the system are given in the paper. In the specific case $\mathrm{K} \rightarrow \infty$,this model is reduced to J. Cohen's model.

A more general model of a queuing system which is a combination of a multi-channel Erlang model, $\mathrm{M} / \mathrm{M} / \mathrm{m} / \mathrm{E}$ model, and also multi-channel classical model $(\mathrm{M} / \mathrm{M} / \mathrm{m}$ models $)$ is considered in the work of authors [3]. A complete formula derivation for probabilistic characteristics, and also for the first and second moments of numerical and temporary characteristics of this type of aqueuing systemis presented in work [7]; a general algorithm of queuing models mathematical formalization taken from monographs [4] and [5] is used.

A mathematical model of an open multi-channel system of queuing having $m$ service facilities of identical efficiency with exponentially distributed service time is presented in this paper. A demand input stream in this case is a superposition of components'random number $h$, each of which represents a Poisson stream of demands served in the order of arrival. For each type of demands entering the system from the $\mathrm{j}$-th source there is a specific size-limited queue $\varepsilon_{j}$ where $\varepsilon_{0}<\varepsilon_{1}<\varepsilon_{2}<\cdots<\varepsilon_{h}$.

A zero (Erlang) component contains demands which are served only if there is at least one free service facility, and they never stand in a queue. In the case, if at the time when the next similar demand arrives in the system there is no free service facility this demand is refused and leaves the system unserved. The model of a queuing system, containing one such component in an input stream, is the Erlang model; therefore we will call this component an Erlang component.

The first component includes demands which are served if there is a free service facility, or they stand in a queue if the number of demands in the queue is fewer than a particular number $\varepsilon_{1}$. In case when there is already available $\varepsilon_{1}$ or more demands in a queue, a newly arrived demand from the first source is refused and leaves the system unserved.

The second component contains demands which are served if there is a free 
service facility, or they stand in a queue if the number of demands in a queue is fewer than a particular number $\varepsilon_{2}>\varepsilon_{1}$. In the case when $\varepsilon_{2}$ or more demands are already available in the queue, an arrived demand from this source is refused and leaves the system unserved, and so on.

In general, the h-th component includes demands which are served if there is a free service facility, or they stand in a queue if the number of demands in the queue are fewer than a particular number $\varepsilon_{h}>\varepsilon_{h-1}>\cdots>\varepsilon_{1}$. In case when there are already $\varepsilon_{h}$ demands in the queue, a newly arrived demand from the h-th source is refused and leaves the system unserved.

Let us accept the following designations:

$$
\varepsilon_{0}=E_{0}=0 ; \quad \varepsilon_{1}=E_{1} ; \quad \varepsilon_{2}=E_{1}+E_{2} ; \cdots \varepsilon_{j}=\sum_{i=0}^{j} E_{i}=\sum_{i=1}^{j} E_{i}
$$

a size-limited queue (memory volume) for demands of the $\mathrm{j}$-th component;

$$
\Lambda_{0}=\sum_{j=0}^{h} \lambda_{j} ; \quad \Lambda_{1}=\sum_{j=1}^{h} \lambda_{j} ; \quad \Lambda_{2}=\sum_{j=2}^{h} \lambda_{j} ; \cdots \Lambda_{h}=\lambda_{h}
$$

where $\lambda_{j}$ demand stream intensity of the $\mathrm{j}$-th component;

$$
R_{0}=\sum_{j=0}^{h} \rho_{j} ; \quad R_{1}=\sum_{j=1}^{h} \rho_{j} ; \quad R_{2}=\sum_{j=2}^{h} \rho_{j} ; \cdots R_{h}=\rho_{h} ; \quad R_{i}=\frac{\Lambda_{i}}{\mu}
$$

where $\rho_{j}$ is the given demand stream intensity of the $\mathrm{j}$-th component.

Demand streams arriving from each source are Poisson and have intensity $\lambda_{j}$; in this case total streams with intensities $\Lambda_{j}$ also have, as we know, a Poisson character. Let us designate the mean intensity of demand service by one service facility as $\mu$. In this case the intensity of an output stream of served demands before the $m$-th states is multiple $\mu$ and depends on the number of busy channels. After the $m$-th state the intensity of served demand stream is equal to $m \mu$. The served demand stream is also Poisson.

With accepted designations and assumptions taken into account, we will obtain a continuous-time Markov chain. 


\section{Probabilistic Characteristics of a Queuing System in a Steady-State Mode}

We make up a set of Kolmogorov-Chapman equations for probabilities of QS states in a steady-state mode of its functioning. Adding the normalization condition $\sum_{i=0}^{m+\varepsilon_{h}} P_{i}=1$, to this set of equations, we obtain a system

$$
\left\{\begin{array}{l}
P_{0} \Lambda_{0}=P_{1} \mu \\
P_{1} \Lambda_{0}+P_{1} \mu=P_{0} \Lambda_{0}+P_{2} 2 \mu \\
P_{2} \Lambda_{0}+P_{2} 2 \mu=P_{1} \Lambda_{0}+P_{3} 3 \mu ; \\
\vdots \\
P_{m-1} \Lambda_{0}+P_{m-1}(m-1) \mu=P_{m-2} \Lambda_{0}+P_{m} m \mu ; \\
P_{m} \Lambda_{1}+P_{m} m \mu=P_{m-1} \Lambda_{0}+P_{m+1} m \mu ; \\
P_{m+1} \Lambda_{1}+P_{m+1} m \mu=P_{m} \Lambda_{1}+P_{m+2} m \mu ; \\
\vdots \\
P_{m+\varepsilon_{1}-1} \Lambda_{1}+P_{m+\varepsilon_{1}-1} m \mu=P_{m+\varepsilon_{1}-2} \Lambda_{1}+P_{m+\varepsilon_{1}} m \mu ; \\
P_{m+\varepsilon_{1}} \Lambda_{2}+P_{m+\varepsilon_{1}} m \mu=P_{m+\varepsilon_{1}-1} \Lambda_{1}+P_{m+\varepsilon_{1}+1} m \mu ; \\
P_{m+\varepsilon_{1}+1} \Lambda_{2}+P_{m+\varepsilon_{1}+1} m \mu=P_{m+\varepsilon_{1}} \Lambda_{2}+P_{m+\varepsilon_{1}+2} m \mu ; \\
\vdots \\
P_{m+\varepsilon_{2}-1} \Lambda_{2}+P_{m+\varepsilon_{2}-1} m \mu=P_{m+\varepsilon_{2}-2} \Lambda_{2}+P_{m+\varepsilon_{2}} m \mu ; \\
P_{m+\varepsilon_{2}} \Lambda_{3}+P_{m+\varepsilon_{2}} m \mu=P_{m+\varepsilon_{2}-1} \Lambda_{2}+P_{m+\varepsilon_{2}+1} m \mu ; \\
P_{m+\varepsilon_{2}+1} \Lambda_{3}+P_{m+\varepsilon_{2}+1} m \mu=P_{m+\varepsilon_{2}} \Lambda_{3}+P_{m+\varepsilon_{2}+2} m \mu ; \\
\vdots \\
P_{m+\varepsilon_{h}-1} \Lambda_{h}+P_{m+\varepsilon_{h}-1} m \mu=P_{m+\varepsilon_{h}-2} \Lambda_{h}+P_{m+\varepsilon_{h}} m \mu ; \\
P_{m+\varepsilon_{h}} m \mu=P_{m+\varepsilon_{h}-1} \Lambda_{h} ; \\
m+\varepsilon_{h} \\
\sum_{i=0} P_{i}=1,
\end{array}\right.
$$

that has a unique solution

$$
\begin{aligned}
P_{0}=\left[e_{m}\left(R_{0}\right)+\frac{R_{0}^{m}}{m !} \sum_{g=1}^{h}\right. & \prod_{j=0}^{g-1}\left(\frac{R_{j}}{m}\right)^{E_{j}} \\
& \times\left\{\begin{array}{l}
\frac{R_{g}}{m-R_{g}}\left(1-\left(\frac{R_{g}}{m}\right)^{E_{g}}\right), R_{g} \neq m \\
E_{g}, \quad R_{g}=m
\end{array}\right\},
\end{aligned}
$$




$$
P_{i}=\left\{\begin{array}{l}
\frac{R_{0}^{i}}{i !} P_{0}, \quad 0<i \leq m, \\
\left(\frac{R_{j+1}}{m}\right)^{i-m-\varepsilon_{j}} \prod_{g=0}^{j}\left(\frac{R_{g}}{m}\right)^{E_{g}} \frac{R_{0}^{m}}{m !} P_{0}, \quad m+\varepsilon_{j} \leq i \leq m+\varepsilon_{j+1}, \\
0 \leq j \leq h-1,
\end{array}\right.
$$

where the designation [1] $e_{m}\left(R_{0}\right)=\sum_{i=0}^{m} \frac{R_{0}^{i}}{i !}$ is accepted - a non-complete exponential function. The solution (1)-(2) defines expressions for probabilities of all possible QS states of this type in a steady-state mode of its functioning.

For further calculations it is convenient to introduce the following basic probabilistic characteristics of QS of this type through which all other quantities are expressed:

- basic probability 1:

$$
P_{B 1}=\sum_{i=m}^{m+\varepsilon_{1}-1} P_{i}=\frac{1-\left(\frac{R_{1}}{m}\right)^{E_{1}}}{1-\frac{R_{1}}{m}} \frac{R_{0}^{m}}{m !} P_{0}
$$

- basic probability 2 :

$$
P_{B 2}=\sum_{i=m+\varepsilon_{1}}^{m+\varepsilon_{2}-1} P_{i}=\frac{1-\left(\frac{R_{2}}{m}\right)^{E_{2}}}{1-\frac{R_{2}}{m}}\left(\frac{R_{1}}{m}\right)^{E_{1}} \frac{R_{0}^{m}}{m !} P_{0}
$$

— basic probability h:

$$
P_{B h}=\sum_{i=m+\varepsilon_{h-1}}^{m+\varepsilon_{h}-1} P_{i}=\frac{1-\left(\frac{R_{h}}{m}\right)^{E_{h}}}{1-\frac{R_{h}}{m}} \prod_{g=1}^{h-1}\left(\frac{R_{g}}{m}\right)^{E_{g}} \frac{R_{0}^{m}}{m !} P_{0}
$$

- congestion probability of the system:

$$
P_{m+\varepsilon_{h}}=\prod_{g=1}^{h}\left(\frac{R_{g}}{m}\right)^{E_{g}} \frac{R_{0}^{m}}{m !} P_{0} .
$$

As a result, a general formula for basic probability is written in the form

$$
P_{B i}=\prod_{g=0}^{i-1}\left(\frac{R_{g}}{m}\right)^{E_{g}} \frac{R_{0}^{m}}{m !} P_{0}\left\{\begin{array}{l}
\frac{m}{m-R_{i}}\left(1-\left(\frac{R_{i}}{m}\right)^{E_{i}}\right), \quad R_{i} \neq m \\
E_{i}, \quad R_{i}=m
\end{array} .\right.
$$


By means of the expression (4) it is possible to present traditional probabilistic characteristics of a queuing system in the most compact form:

- probability of a newly arrived demand service expectation in the queue

$$
\begin{aligned}
P_{W}=\frac{\Lambda_{1}}{\Lambda_{0}} \sum_{i=m}^{m+\varepsilon_{1}-1} P_{i}+\frac{\Lambda_{2}}{\Lambda_{0}} \sum_{i=m+\varepsilon_{1}}^{m+\varepsilon_{2}-1} P_{i} & +\frac{\Lambda_{3}}{\Lambda_{0}} \sum_{i=m+\varepsilon_{2}}^{m+\varepsilon_{3}-1} P_{i} \\
& +\cdots+\frac{\Lambda_{h}}{\Lambda_{0}} \sum_{i=m+\varepsilon_{h-1}}^{m+\varepsilon_{h}-1} P_{i}=\frac{1}{R_{0}} \sum_{i=1}^{h} R_{i} P_{B i}
\end{aligned}
$$

- probability of a newly arrived demand service refusal (probability of demand loss)

$$
\begin{gathered}
P_{L}=\frac{\Lambda_{0}-\Lambda_{1}}{\Lambda_{0}} \sum_{i=m}^{m+\varepsilon_{1}-1} P_{i}+\frac{\Lambda_{0}-\Lambda_{2}}{\Lambda_{0}} \sum_{i=m+\varepsilon_{1}}^{m+\varepsilon_{2}-1} P_{i}+\frac{\Lambda_{0}-\Lambda_{3}}{\Lambda_{0}} \sum_{i=m+\varepsilon_{2}}^{m+\varepsilon_{3}-1} P_{i} \\
+\cdots+\frac{\Lambda_{0}-\Lambda_{h}}{\Lambda_{0}} \sum_{i=m+\varepsilon_{h-1}}^{m+\varepsilon_{h}-1} P_{i}+P_{m+\varepsilon_{h}}=\frac{1}{R_{0}} \sum_{i=1}^{h}\left(R_{0}-R_{i}\right) P_{B i}+P_{m+\varepsilon_{h}} \\
=\sum_{i=1}^{h} P_{B i}-P_{W}+P_{m+\varepsilon_{h}}=1-P_{I S}-P_{W} .
\end{gathered}
$$

The probability of an immediate service of a newly arrived demand has, apparently, a form

$$
P_{I S}=\sum_{i=0}^{m-1} P_{i}=e_{m-1}\left(R_{0}\right) P_{0}
$$

\section{Numerical Characteristics of a Queuing System}

By means of probabilistic characteristics of the system found above, it is possible to express all main features characterizing a steady-state mode of a queuing system functioning. So, through put capacity of a queuing system is a number of demands passing through the system per unit of time $A=\Lambda_{0} q=\Lambda_{0}\left(1-P_{L}\right)=$ $\Lambda_{0}\left(P_{I S}+P_{W}\right)$. This number includes all demands from a general input stream except refused demands and those that did not get into the system. Relative through put capacity of the system, thus, is a share of demands passing through a queuing system from a general input stream of demands $q=1-P_{L}$. 
The average number of demands under service at the same time (or, that is the same, an average number of busy channels) with formulae (2) - (5) taken into account has a form

$$
\begin{aligned}
\bar{n}=\sum_{i=1}^{m-1} i P_{i}+m \sum_{i=m}^{m+\varepsilon_{h}} P_{i}=R_{0} P_{0} e_{m-2}\left(R_{0}\right)+m\left(P_{W}+P_{L}\right) \\
=R_{0} P_{0} e_{m-2}\left(R_{0}\right)+m\left(\sum_{i=1}^{h} P_{B i}+P_{m+\varepsilon_{h}}\right) .
\end{aligned}
$$

The second initial moment of demands number under service is

$$
\begin{aligned}
\overline{n^{2}}=\sum_{i=1}^{m-1} i^{2} P_{i} & +m^{2} \sum_{i=m}^{m+\varepsilon_{h}} P_{i} \\
& =R_{0} P_{0} e_{m-2}\left(R_{0}\right)+R_{0}^{2} P_{0} e_{m-3}\left(R_{0}\right)+m^{2}\left(\sum_{i=1}^{h} P_{B i}+P_{m+\varepsilon_{h}}\right)
\end{aligned}
$$

An average demands number in a queue (average queue length) are

$$
\begin{aligned}
\bar{l}=\sum_{i=m+1}^{m+\varepsilon_{h}}(i-m) P_{i} & \\
& =\sum_{i=1}^{h}\left\{\begin{array}{l}
\frac{R_{i}}{m-R_{i}}\left[P_{B i}-E_{i} P_{m+\varepsilon_{i}}\right], \quad R_{i} \neq m \\
\frac{E_{i}\left(E_{i}+1\right)}{2} P_{m+\varepsilon_{i-1}}, \quad R_{i}=m
\end{array}\right\}+\sum_{i=2}^{h} \varepsilon_{i-1} \frac{R_{i}}{m} P_{B i} .
\end{aligned}
$$

The second initial moment of demands number in a queue is

$$
\begin{aligned}
& \overline{l^{2}}=\sum_{i=m+1}^{m+\varepsilon_{h}}(i-m)^{2} P_{i} \\
& =\sum_{i=1}^{h}\left[\varepsilon_{i-1}^{2} P_{B i}+\left\{\begin{array}{l}
\frac{R_{i}}{m-R_{i}}\left(\frac{m+R_{i}}{m-R_{i}}+2 \varepsilon_{i-1}\right) P_{B i}- \\
-\frac{m E_{i}}{m-R_{i}}\left(E_{i}+\frac{2 R_{i}}{m-R_{i}}+2 \varepsilon_{i-1}\right) P_{m+\varepsilon_{i}}, R_{i} \neq m \\
\left(E_{i}-1\right) E_{i}\left(\frac{2 E_{i}-1}{6}+\varepsilon_{i-1}\right) P_{m+\varepsilon_{i}}, \quad R_{i}=m
\end{array}\right\}\right\rfloor \\
& +\varepsilon_{h}^{2} P_{m+\varepsilon_{h}} .
\end{aligned}
$$

An average demands number in the system on the whole (both in a queue and under service) are 


$$
\begin{aligned}
\bar{k}=\bar{n}+\bar{l}=R_{0} & P_{0} e_{m-2}\left(R_{0}\right) \\
+ & +\sum_{i=1}^{h}\left[\begin{array}{r}
\frac{R_{i}}{m-R_{i}}\left[P_{B i}-E_{i} P_{m+\varepsilon_{i}}\right], \quad R_{i} \neq m \\
\frac{E_{i}\left(E_{i}+1\right)}{2} P_{m+\varepsilon_{i-1}}, \quad R_{i}=m
\end{array}\right\} \\
& \left.+\left(m+\varepsilon_{i-1} \frac{R_{i}}{m}\right) P_{B i}\right]+m P_{m+\varepsilon_{h}} .
\end{aligned}
$$

The covariance of demands number under service and the number of demands in a queue is

$$
\begin{aligned}
K_{n l} & =\overline{n l}-\bar{n} \bar{l} \\
& =\sum_{i=m+1}^{\infty} m(i-m) P_{i}-\bar{n} \bar{l} \\
& =m \sum_{i=m+1}^{\infty}(i-m) P_{i}-\bar{n} \bar{l} \\
& =m \bar{l}-\bar{n} \bar{l} .
\end{aligned}
$$

Therefore, the second initial moment of a total demands number in the system is

$$
\begin{aligned}
& \overline{k^{2}}=\overline{n^{2}}+\overline{l^{2}}+ 2 m \bar{l}=R_{0} P_{0} e_{m-2}\left(R_{0}\right)+R_{0}^{2} P_{0} e_{m-3}\left(R_{0}\right) \\
&+\sum_{i=1}^{h}\left[\begin{array}{l}
\frac{R_{i}}{m-R_{i}}\left(\frac{m+R_{i}}{m-R_{i}}+2 \varepsilon_{i-1}+2 m\right) P_{B i}- \\
E_{i}\left[\left(E_{i}-1\right)\left(\frac{2 E_{i}-1}{6}+\varepsilon_{i-1}\right)+\right.
\end{array}\right. \\
& \quad-\frac{m E_{i}}{m-R_{i}}\left(E_{i}+\frac{2 R_{i}}{m-R_{i}}+2 \varepsilon_{i-1}+2 R_{i}\right) P_{m+\varepsilon_{i}}, \quad R_{i} \neq m \\
&\left.+\left(E_{i}+1\right) m\right] \begin{array}{l}
P_{m+\varepsilon_{i}}, \quad R_{i}=m \\
\left.\quad+\left(\varepsilon_{i-1}^{2}+2 \varepsilon_{i-1} R_{i}+m^{2}\right) P_{B i}\right]+\left(\varepsilon_{h}^{2}+m^{2}\right) P_{m+\varepsilon_{h}} .
\end{array}
\end{aligned}
$$

Further, in the considered queuing system,the queue is possible only when all service facilities are busy. Thus, the total stream of served demands of the whole system consists of service streams of each channel and has $m \mu$ intensity. In this case, the probability that the system serves $i$ demands during $t$ time in the event of queue, will be recorded in the form $B_{i}(t)=\frac{(m \mu t)^{i}}{i !} e^{-m \mu t}$.

The function of service waiting time distribution for one demand we will find according to a known dependence $F_{W}(t)=1-P\left(t_{W} \geq t\right)$, where $P\left(t_{W} \geq t\right)$ - the probability that waiting time in a queue for one demand is more than an advanced set time $t$. As it is easy to see, it is possible, firstly, in case when the 
queue is absent, but a newly arrived demand finds all service facilities in the system busy, and during $t$ time none of facilities is released. Secondly, in case when one demand is already in a queue and during $t$ time the system serves no more than one demand, or there are two demands in a queue, and during $t$ time no more than two demands are served, and so on. In this case, according to the formula of full probability, we have

$$
\begin{gathered}
q\left[1-F_{W}(t)\right]= \\
=\frac{\Lambda_{1}}{\Lambda}\left[B_{0}(t) \sum_{i=m}^{m+\varepsilon_{1}-1} P_{i}+B_{1}(t) \sum_{i=m+1}^{m+\varepsilon_{1}-1} P_{i}+\cdots+\right. \\
\left.B_{\varepsilon_{1}-1}(t) P_{m+\varepsilon_{1}-1}\right]+ \\
+\frac{\Lambda_{2}}{\Lambda}\left[\sum_{i=0}^{\varepsilon_{1}} B_{i}(t) \sum_{i=m+\varepsilon_{1}}^{m+\varepsilon_{2}-1} P_{i}+B_{\varepsilon_{1}+1}(t) \sum_{i=m+\varepsilon_{1}+1}^{m+\varepsilon_{2}-1} P_{i}+\cdots+\right. \\
+\frac{\Lambda_{3}}{\Lambda}\left[\sum_{i=0}^{\varepsilon_{2}} B_{i}(t) \sum_{i=m+\varepsilon_{2}-1}^{m+\varepsilon_{3}-1} P_{i}+B_{\varepsilon_{2}+1}(t) \sum_{\left.i=m+\varepsilon_{2}-1\right]+\varepsilon_{2}+1}^{m+\varepsilon_{3}-1} P_{i}+\cdots+\right. \\
+\frac{\Lambda_{h}}{\Lambda}\left[\sum_{i=0}\left[B_{\varepsilon_{3}-1}(t) P_{m+\varepsilon_{3}-1}\right]+\cdots+\right. \\
\sum_{i=m+\varepsilon_{h-1}} B_{i}(t) P_{\varepsilon_{h}}^{m+\varepsilon_{h}-1} \\
\left.+B_{\varepsilon_{h}-1}(t) P_{m+\varepsilon_{h}-1}\right]
\end{gathered}
$$

After a number of intermediate calculations, it is possible to obtain the following expressions for finite-sums sequence in square brackets in the righthand side of this ratio:

$$
B_{0}(t) \sum_{i=m}^{m+\varepsilon_{1}-1} P_{i}+B_{1}(t) \sum_{i=m+1}^{m+\varepsilon_{1}-1} P_{i}+\cdots+B_{\varepsilon_{1}-1}(t) P_{m+\varepsilon_{1}-1}=
$$




$$
\begin{aligned}
& =e^{-m \mu t} \frac{R^{m}}{m !} P_{0} \frac{1}{1-\frac{R_{1}}{m}}\left[e_{\varepsilon_{1}-1}\left(R_{1} \mu t\right)-\left(\frac{R_{1}}{m}\right)^{E_{1}} e_{\varepsilon_{1}-1}(m \mu t)\right] . \\
& \sum_{i=0}^{\varepsilon_{1}} B_{i}(t) \sum_{i=m+\varepsilon_{1}}^{m+\varepsilon_{2}-1} P_{i}+B_{\varepsilon_{1}+1}(t) \sum_{i=m+\varepsilon_{1}+1}^{m+\varepsilon_{2}-1} P_{i}+\cdots+B_{\varepsilon_{2}-1}(t) P_{m+\varepsilon_{2}-1}= \\
& =e^{-m \mu t} \frac{R^{m}}{m !} P_{0} \frac{1}{1-\frac{R_{2}}{m}}\left[\left(\frac{R_{1}}{m}\right)^{E_{1}} e_{\varepsilon_{1}-1}(m \mu t)+\right. \\
& +\left(\frac{R_{1}}{R_{2}}\right)^{E_{1}}\left[e_{\varepsilon_{2}-1}\left(R_{2} \mu t\right)-e_{\varepsilon_{1}-1}\left(R_{2} \mu t\right)\right]- \\
& \left.-\left(\frac{R_{1}}{m}\right)^{E_{1}}\left(\frac{R_{2}}{m}\right)^{E_{2}} e_{\varepsilon_{2}-1}(m \mu t)\right] . \\
& \sum_{i=0}^{\varepsilon_{2}} B_{i}(t) \sum_{i=m+\varepsilon_{2}}^{m+\varepsilon_{3}-1} P_{i}+B_{\varepsilon_{2}+1}(t) \sum_{i=m+\varepsilon_{2}+1}^{m+\varepsilon_{3}-1} P_{i}+\cdots+B_{\varepsilon_{3}-1}(t) P_{m+\varepsilon_{3}-1}= \\
& =e^{-m \mu t} \frac{R^{m}}{m !} P_{0} \frac{1}{1-\frac{R_{3}}{m}}\left[\left(\frac{R_{1}}{m}\right)^{E_{1}}\left(\frac{R_{2}}{m}\right)^{E_{2}} e_{\varepsilon_{2}-1}(m \mu t)+\right. \\
& +\left(\frac{R_{1}}{R_{3}}\right)^{E_{1}}\left(\frac{R_{2}}{R_{3}}\right)^{E_{2}}\left[e_{\varepsilon_{3}-1}\left(R_{3} \mu t\right)-e_{\varepsilon_{2}-1}\left(R_{3} \mu t\right)\right]- \\
& \left.-\left(\frac{R_{1}}{m}\right)^{E_{1}}\left(\frac{R_{2}}{m}\right)^{E_{2}}\left(\frac{R_{3}}{m}\right)^{E_{3}} e_{\varepsilon_{3}-1}(m \mu t)\right] .
\end{aligned}
$$

and so on; thus in general we have

$$
\begin{gathered}
\sum_{i=0}^{\varepsilon_{h-1}} B_{i}(t) \sum_{i=m+\varepsilon_{h-1}}^{m+\varepsilon_{h}-1} P_{i}+B_{\varepsilon_{h-1}+1}(t) \sum_{i=m+\varepsilon_{h-1}+1}^{m+\varepsilon_{h}-1} P_{i}+\cdots+ \\
+B_{\varepsilon_{h}-1}(t) P_{m+\varepsilon_{h}-1}= \\
=e^{-m \mu t} \frac{R^{m}}{m !} P_{0} \frac{1}{1-\frac{R_{h}}{m}}\left[\prod_{g=1}^{h-1}\left(\frac{R_{g}}{m}\right)^{E_{g}} e_{\varepsilon_{h-1}-1}(m \mu t)+\right.
\end{gathered}
$$




$$
\begin{gathered}
+\prod_{g=1}^{h-1}\left(\frac{R_{g}}{R_{h}}\right)^{E_{g}}\left[e_{\varepsilon_{h}-1}\left(R_{h} \mu t\right)-e_{\varepsilon_{h-1}-1}\left(R_{h} \mu t\right)\right]- \\
\left.-\prod_{g=1}^{h}\left(\frac{R_{g}}{m}\right)^{E_{g}} e_{\varepsilon_{h}-1}(m \mu t)\right\rfloor
\end{gathered}
$$

As a result, substituting ratios obtained above (7) - (10) into the right member of a formula (6), we will finally find

$$
\begin{aligned}
& F_{W}(t)=1-e^{-m \mu t} \frac{P_{m-1}}{q}+ \\
& +\left\{\frac{R_{1}}{m-R_{1}}\left[e_{\varepsilon_{1}-1}\left(R_{1} \mu t\right)-\left(\frac{R_{1}}{m}\right)^{E_{1}} e_{\varepsilon_{1}-1}(m \mu t)\right]+\right. \\
& +\sum_{i=2}^{h} \frac{R_{i}}{m-R_{i}}\left[\prod_{g=1}^{i-1}\left(\frac{R_{g}}{m}\right)^{E_{g}} e_{\varepsilon_{i-1}-1}(m \mu t)+\right. \\
& \prod_{g=1}^{i-1}\left(\frac{R_{g}}{R_{i}}\right)^{E_{g}}\left[e_{\varepsilon_{i}-1}\left(R_{i} \mu t\right)-e_{\varepsilon_{i-1}-1}\left(R_{i} \mu t\right)\right]- \\
& \left.\left.-\prod_{g=1}^{i}\left(\frac{R_{g}}{m}\right)^{E_{g}} e_{\varepsilon_{i}-1}(m \mu t)\right\rfloor\right\}
\end{aligned}
$$

Hence, the density of a demand waiting time distribution for service in a queue is

$$
\begin{gathered}
f_{W}(t)=\frac{d F_{W}(t)}{d t}=e^{-m \mu t} \frac{P_{m-1}}{q} \times \\
\times\left\{\Lambda_{1} e_{\varepsilon_{1}-1}\left(\Lambda_{1} t\right)+\sum_{i=2}^{h} \Lambda_{i} \prod_{g=1}^{i-1}\left(\frac{R_{g}}{R_{i}}\right)^{E_{g}}\left[e_{\varepsilon_{i}-1}\left(\Lambda_{i} t\right)-e_{\varepsilon_{i-1}-1}\left(\Lambda_{i} t\right)\right]\right\}
\end{gathered}
$$

and then, mean waiting time of demand service in a queue is

$$
\bar{t}_{W}=\int_{0}^{\infty} t f_{W}(t) d t=
$$




$$
=\frac{1}{\Lambda_{0} q} \sum_{i=1}^{h}\left\{\frac{R_{i}}{m-R_{i}}\left[P_{B i}-E_{i} P_{m+\varepsilon_{i}}\right]+\frac{R_{i}}{m} \varepsilon_{i-1} P_{B i}\right\}=\frac{\bar{l}}{A}
$$

in compliance with J. Littl's formulae. In the same way the second initial moment of a demand waiting time in a queue is

$$
\begin{gathered}
\overline{t_{W}^{2}}=\int_{0}^{\infty} t^{2} f_{W}(t) d t= \\
=\frac{1}{\Lambda_{0} q} \sum_{i=1}^{h} R_{i}\left\{\begin{array}{l}
\frac{2\left(P_{B i}-E_{i} P_{m+\varepsilon_{i}}\right)}{\mu\left(m-R_{i}\right)^{2}}\left[1+\frac{\varepsilon_{i-1}}{m}\left(m-R_{i}\right)\right]+ \\
\frac{P_{m}}{3 m^{2} \mu} \prod_{g=0}^{i-1}\left(\frac{R_{g}}{R_{i}}\right)^{E_{g}} \times \\
+\frac{\varepsilon_{i-1}\left(\varepsilon_{i-1}+1\right) P_{B i}}{m^{2} \mu}-\frac{E_{i}\left(E_{i}+1\right) P_{m+\varepsilon_{i}}}{m \mu\left(m-R_{i}\right)}, \quad R_{i} \neq m \\
\times\left[\varepsilon_{i}\left(\varepsilon_{i}+1\right)\left(\varepsilon_{i}+2\right)-\varepsilon_{i-1}\left(\varepsilon_{i-1}+1\right)\left(\varepsilon_{i-1}+2\right)\right], \quad R_{i}=m
\end{array}\right\} .
\end{gathered}
$$

Mean sojourn time of a demand on the whole (both in a queue, and under service) is, apparently,

$$
\begin{gathered}
\bar{t}_{T}=\bar{t}_{S}+\bar{t}_{W}=\frac{1}{\Lambda_{0} q}\left\{R_{0} P_{I S}+\sum_{i=1}^{h}\left[\frac{R_{i}}{m-R_{i}}\left(P_{B i}-E_{i} P_{m+\varepsilon_{i}}\right)+\right.\right. \\
\left.\left.+\frac{R_{i}}{m}\left(\varepsilon_{i-1}+m\right) P_{B i}\right]\right\}=\frac{\bar{k}}{A}
\end{gathered}
$$

and then, the second initial moment of a demand sojourn time is

$$
\begin{gathered}
\overline{t_{T}^{2}}=\overline{t_{W}^{2}}+\overline{t_{S}^{2}}+2 \bar{t}_{W} \bar{t}_{S}= \\
=\frac{1}{\mu \Lambda_{0} q}\left\{2 R_{0} P_{I S}+\sum_{i=1}^{h} R_{i}\left[\frac{2\left(P_{B i}-E_{i} P_{m+\varepsilon_{i}}\right)}{\left(m-R_{i}\right)^{2}} \times\right.\right. \\
\times\left[1+\left(\frac{\varepsilon_{i-1}}{m}+1\right)\left(m-R_{i}\right)\right]+ \\
\left.\left.+\left[2+\frac{\varepsilon_{i-1}}{m}\left(\frac{\varepsilon_{i-1}+1}{m}+2\right)\right] P_{B i}-\frac{E_{i}\left(E_{i}+1\right) P_{m+\varepsilon_{i}}}{m\left(m-R_{i}\right)}\right]\right\} .
\end{gathered}
$$


Let us note that the ratio (11) gives a possibility to calculate moments of any order as a demand waiting time in a queue for service, and demand sojourn time in general (in a queue and under service). The model presented in this paper is the most general in relation to earlier studied models [2], [6] and [3].

\section{References}

[1] Handbook of Mathematical Functions with Formulas, Graphs and Mathematical Tables, ed. by M. Abramowitz and I. A. Stegun, Dover Publications, New York (1970).

[2] J.W. Cohen, Certain delay problems for a full availability trunk group loaded by two sources, Communication News, 16, No. 3 (1956), 105-113.

[3] A.P. Kirpichnikov, A.S. Titovtsev, Open systems of multicomponent flows differentiated service, Ciência e Técnica Vitivinícola, 29, No. 7 (2014), 108-122.

[4] A.P.Kirpichnikov Prikladnaya teoriya massovogo obsluzhivaniya [Applied queuing theory], Publishing office of KSU Publ., Kazan (2008), In Russian.

[5] A.P.Kirpichnikov Metody prikladnoy teorii massovogo obsluzhivaniya [Methods of applied queuing theory], Publishing office of KSU Publ., Kazan (2011), In Russian.

[6] H. Takagi, Explicit delay distribution in first-come first-served $M / M / m / K$ and $\mathrm{M} / \mathrm{M} / \mathrm{m} / \mathrm{K} / \mathrm{n}$ queues and mixed loss-delay system, International Journal of Pure and Applied Mathematics, 40, No. 2 (2007), 185-200.

[7] A. Titovtsev Sistemy differentsirovannogo obsluzhivaniya polikomponentnykh potokov. Modeli i kharakteristiki [Systems of differentiated services multicomponent flows. Models and specifications], LAP LAMBERT Academic Publishing GmbH \& Co. KG Publ., Saarbrücken (2012), In Russian. 
